\title{
Mitteilungen des Arbeitskreises der Chefärzte und Chef- ärztinnen von Kliniken für Psychiatrie und Psychotherapie an Allgemeinkrankenhäusern in Deutschland (ackpa)
}

Verantwortlich für diese Rubrik: Karl H. Beine, Hamm

\section{Seit 30 Jahren: Die Kommende-Tagung $\nabla$}

Wer einen gedruckten Hinweis auf die Kommende-Tagung vor sich hat und nicht bereits gehört hat, wovon die Rede ist, geht in der Regel davon aus, es handele sich um die Ankündigung einer kommenden Tagung. Die Betonung ist jedoch auf der 2. Silbe, also Komménde-Tagung. Wie das? Die Bezeichnung geht auf die Kommende zurück, den ersten Tagungsort in Dortmund-Brakel, ehemals Sitz des Deutschen Ritterordens und heutzutage eine Bildungseinrichtung, wo die halbjährlichen Treffen ab 1985 stattfanden.

Die Differenz zwischen Gesprochenem und Geschriebenem begleitet die Kommende-Tagung: Die Tagungseinladungen von damals sind nicht archiviert; das, was die Kommende-Tagung ist, findet sich zumindest für die weiter zurückliegenden Jahre vor der Jahrtausendwende nicht in Dokumenten und schriftlichen Veröffentlichungen, sondern mehr über „oral history“: Diejenigen, die dabei waren, können am besten erzählen, wer da war und was vorgetragen und diskutiert wurde.

In der Kommende trafen sich seit 1985 auf Einladung von Dr. Hanns Philipzen, Chefarzt der Psychiatrischen Klinik in Bad Driburg, die Leiter von psychiatrischen Kliniken an Allgemeinkrankenhäusern mit Pflichtversorgung für einen definierten psychiatrischen Sektor. Die Tagungen beschäftigten sich insbesondere mit der Frage: Was braucht der Sektor?

Die stationäre psychiatrische Versorgung in wohnortfernen Großkrankenhäusern mit Langzeitstationen war nach der Psychiatrie-Enquête von 1975 infrage gestellt: Psychiatrische Kliniken am Allgemeinkrankenhaus traten zunehmend mit dem Anspruch an, die Pflicht- und Vollversorgung eines Sektors zu übernehmen. Um nichts weniger ging es den Gründervätern des Arbeitskreises: Die Verzichtbarkeit von Großkrankenhäusern praktisch zu beweisen, indem sie die Vollver- sorgung für einen definierten Sektor übernahmen und keine Patienten in Großkrankenhäuser verlegten. Der Beweis ist mittlerweile längst erbracht: Psychiatrische Kliniken gewährleisten heutzutage selbstverständlich die Vollversorgung eines Sektors.

Die Kommende war pragmatisch, solidarisch, parteiisch und engagiert. Die Mitglieder verfolgten ihren Weg konsequent und überzeugt auch gegen Widerstand. Sie waren damals nur wenige, ca. 25 im Jahr 1986 in der BRD.

Daneben steht die Kommende für gemeindepsychiatrische Behandlungs- und Versorgungsprinzipien, über deren Realisierung sich die Klinikleiter verständigten und diskutierten: Psychiatrie mit offenen Türen; Gemeindepsychiatrie in enger Vernetzung mit der Eingliederungshilfe und dem gemeindepsychiatrischen Verbund, dessen Formen und Inhalte damals erst infolge der Enthospitalisierung entwickelt wurden; Sozialpsychiatrie im eigentlichen Sinne, nämlich mit Einbeziehung der Angehörigen und unter Berücksichtigung von Aspekten des Wohnens, Arbeitens und der Freizeit. Die Kommende suchte nach Wegen zur Umsetzung dieser Inhalte in die Praxis und ihre Mitglieder gestalteten sie vor Ort aktiv mit. Wenn ein Chefarzt der Krankenhausverwaltung einen Vorschlag für ein neues Versorgungsprojekt machte und die zweifelnde Frage zurückkam, ob so etwas denn bereits praktisch erprobt sei, konnte er nicht selten auf ein anderes Kommende-Mitglied verweisen - in den damaligen Post-Psychiatrie-Reform-Zeiten ein unschätzbares Plus für gemeindepsychiatrische Projekte, die noch 10 Jahre zuvor undenkbar waren.

Die Gründung von ackpa im Jahr 1983 verfolgte demgegenüber zunächst andere Ziele: Einerseits die politische Speerspitze der Abteilungspsychiatrie zu bilden, andererseits Abteilungen, die noch keine Vollversorgung wahrnahmen, eine Plattform zu bieten - mit dem Ziel, die Leiter letztendlich von der Pflichtversorgung zu überzeugen.

Hanns Philipzen übergab die Leitung und Moderation des Kommende-Arbeitskreises im Jahr 1996/1997 an Dr. Thomas Schulte, Chefarzt der Psychiatrischen Abteilung in Lauterbach. Auf ihn gingen 2 maßgebliche Neuerungen zurück: Der Umzug der Kommende-Tagung nach Kassel ins Ludwig-Noll-Krankenhaus, um den Mitgliedern aus den neuen Bundesländern die Anreise zu verkürzen.

Die zweite Idee, die Dr. Schulte umsetzte, war die Annäherung an ackpa und die Verbindung der Tagungen von Kommende und ackpa.

So findet seit 2001 die KommendeTagung mit ackpa im Frühjahr, die ackpaTagung mit Kommende im Herbst (ab 2015 umgekehrt) statt.

Dr. Schulte übergab die Moderation und Leitung des Kommende-Arbeitskreises im Jahr 2005 an die Autorin. Die „Kommende-Idee“ des praxisnahen, pragmatischen Austauschs und die etwas anarchische, aber hochwirksame Vernetzung von Ideen und Projekten lebt weiter. Die „Schätze“ an Erfahrung und Wissen, die sich im Alltag einer jeden psychiatrischen Klinik ansammeln, werden miteinander geteilt. Powerpoint-Präsentationen sind bis heute nicht Voraussetzung von Kommende-Beiträgen, auch wenn sie von den meisten Vortragenden als praktische Unterstützung genutzt werden. Die vorgetragenen Daten sind nicht unbedingt auf Signifikanz geprüft. Das Berichten, Erzählen und der Austausch hat Vorrang.

Die Kommende trifft sich nun seit 30 Jahren. Dieser kurze geschichtliche Rückblick verdeutlicht: Psychiatriegeschichte wird jeden Moment, an vielen Orten, durch viele Menschen, für viele Menschen gemacht.
Dr. Ingrid Munk
Vivantes Klinikum Neukölln
Rudower Straße 48
12351 Berlin
Ingrid.Munk@vivantes.de 